\title{
Article
}

\section{Carbon nanotubes for stabilization of nanostructured lipid particles}

Gaunt, Nicholas, Patil-Sen, Yogita, Baker, Matthew James and Kulkarni, Chandrashekhar Vishwanath

Available at http://clok.uclan.ac.uk/11352/

Gaunt, Nicholas, Patil-Sen, Yogita, Baker, Matthew James ORCID: 0000-00032362-8581 and Kulkarni, Chandrashekhar Vishwanath ORCID: 0000-00025621-4791 (2015) Carbon nanotubes for stabilization of nanostructured lipid particles. Nanoscale, 7 (3). pp. 1090-1095. ISSN 2040-3364

It is advisable to refer to the publisher's version if you intend to cite from the work. http://dx.doi.org/10.1039/C4NR05593D

For more information about UCLan's research in this area go to http://www.uclan.ac.uk/researchgroups/ and search for <name of research Group>.

For information about Research generally at UCLan please go to http://www.uclan.ac.uk/research/

All outputs in CLoK are protected by Intellectual Property Rights law, including Copyright law. Copyright, IPR and Moral Rights for the works on this site are retained by the individual authors and/or other copyright owners. Terms and conditions for use of this material are defined in the policies page. 


\title{
Nanoscale
}

\section{PAPER}

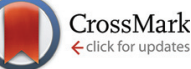

Cite this: Nanoscale, 2015, 7, 1090

\section{Carbon nanotubes for stabilization of nanostructured lipid particles $\uparrow$}

\author{
Nicholas P. Gaunt, ${ }^{a}$ Yogita Patil-Sen, ${ }^{\text {a Matthew J. Baker }}{ }^{\mathrm{a}, \mathrm{b}}$ and \\ Chandrashekhar V. Kulkarni*a
}

\begin{abstract}
Carbon nanotubes (CNTs) are increasingly studied for innovative biotechnological applications particularly where they are combined with essential biological materials like lipids. Lipids have been used earlier for enhancing the dispersibility of CNTs in aqueous solutions. Here we report a novel application of CNTs for stabilization of internally self-assembled nanostructured lipid particles of $2-5 \mu \mathrm{m}$ size. Single-walled (pristine) as well as $-\mathrm{OH}$ and $-\mathrm{COOH}$ functionalized multi-walled CNTs were employed to produce nanostructured emulsions which stayed stable for months and could be re-dispersed after complete dehydration. Concentrations of CNTs employed for stabilization were very low; moreover CNTs were well-decorated with lipid molecules. These features contribute towards reducing their toxicity and improving biocompatibility for biomedical and pharmaceutical applications. Our approach paves the way for future development of combination therapies employing both CNTs and nanostructured lipid self-assembly together as carriers of different drugs.
\end{abstract}

Received 23rd September 2014 Accepted 16th November 2014

DOI: $10.1039 / c 4 n r 05593 d$

www.rsc.org/nanoscale cations, for which the CNT surface needs to be modified by non-covalent or covalent interactions using various molecules like e.g. lipids, proteins, surfactants, drugs, polymers, peptides, nucleic acids and metal nanoparticles. Some of these molecules not only interact but also self-organize on CNT surfaces. ${ }^{13,14}$ Amphiphilic molecules, like lipids, are among such molecules which form self-assembled nanostructures when in solvents. ${ }^{15}$ However, with CNTs they self-organize in such a way that the non-polar part is shielded from polar medium via hydrophobic interactions with CNT surface while the hydrophilic part faces polar solvent medium, which is usually water based. ${ }^{10,13,16-20}$

The type of self-assembled nanostructure, also called liquid crystalline lipid phases interacting with carbon nanotubes could differ from lipid to $\operatorname{lipid}^{21}$ as well as with physicochemical conditions. ${ }^{22}$ Usually the lamellar nanostructure (Fig. 1) is most commonly observed which mimics plasma membrane whereas hexagonal and cubic types of nanostructures resemble complex intracellular biomembranes. ${ }^{15,23}$ Lipid nanostructures are also used for many applications, for example, for separation of biomolecules, ${ }^{24}$ protein crystallization, ${ }^{25}$ synthesis of metal nanoparticles ${ }^{26}$ and electrophoresis gels for amphiphilic molecules. ${ }^{24}$ However, in some cases their applicability is hampered by their extremely high viscosity and inconsistent domain consistency. These problems are resolved by dispersing them into submicron (typical diameter $200-400 \mathrm{~nm}$ ) or micron sized colloidal particles with large quantities of water (about $80-95 \%) .{ }^{27,28}$ The process involves kinetic stabilization of lipid nanostructures using some energy input and stabilizer 


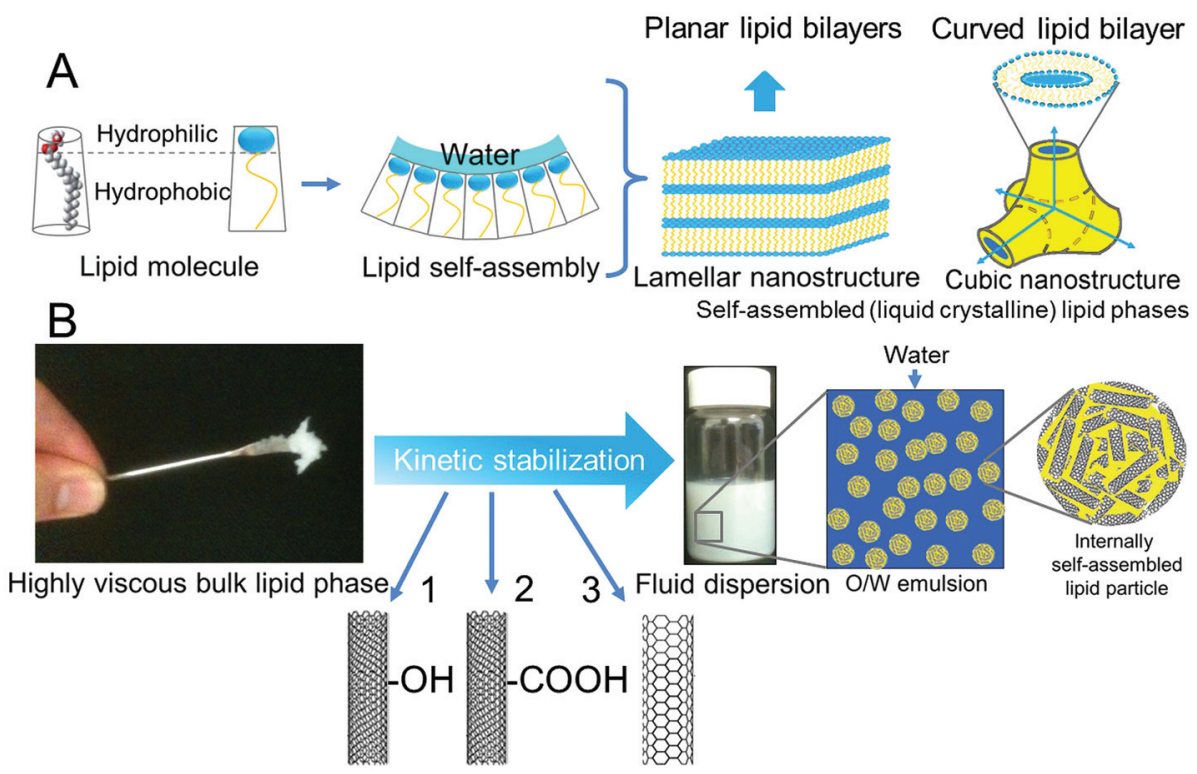

Fig. 1 CNT Stabilized Internally Self-assembled Lipid Particles. (A) In presence of water, amphiphilic lipid molecules self-assemble into various liquid crystalline phases such as lamellar and cubic nanostructures. (B) Highly viscous lipid phases like cubic nanostructures are kinetically stabilized (using ultrasound energy and CNT stabilizer) into fluid dispersion. Pristine single-walled (SW) and functionalized (-OH and $-\mathrm{COOH}$ ) multi-walled (MW) CNTs were used to stabilize the oil-in-water (Pickering) emulsion.

molecules like surfactants, polymers, hydrocolloids or biomolecules resulting into oil-in-water $(\mathrm{O} / \mathrm{W})$ emulsions. ${ }^{28,29}$ (Note: Here 'oil' phase refers to lipid chains region.) The original lipid self-assembly is usually preserved inside dispersed particles and the overall viscosity is reduced by a few orders of magnitudes thereby enhancing their applicability. ${ }^{29,30}$ Major application of internally self-assembled lipid particles is for 'drug delivery' because of the possibility of loading them with hydrophilic, hydrophobic and amphiphilic molecules. ${ }^{31-33}$

In this work we exploit CNTs as novel stabilizers for nanostructured lipid particles as depicted in Fig. 1. Use of CNTs is expected to be significantly advantageous in stabilization of such systems because of their unique surface properties that facilitate extensive functionalization. Presence of lipid also overcame the destabilization of CNTs in aqueous solution which was otherwise occurring in minutes after their dispersion without lipid molecules. As mentioned earlier, CNTs themselves are promising candidates for drug delivery and other biomedical applications. ${ }^{12}$ Combining them with nanostructured lipid particles that could be also loaded with drugs may open up many new pathways for example, for combination therapy or polytherapy i.e. for delivering multiple drugs or a drug and other inhibitory molecules at the same time at the same target. Such therapies are required mainly against multidrug resisting macromolecules or pathogens. ${ }^{34,35}$ The concentration of CNTs required to stabilize an emulsion (per unit volume of water) is extremely low (as low as $0.3 \mu \mathrm{g} \mathrm{ml} \mathrm{m}^{-1}$ ). Furthermore, the biocompatibility of CNTs is expected to be greatly enhanced due to decoration with lipid molecules. These features can be attributed to a very low to negligible in vivo toxicity of CNT stabilized lipid nanoparticles for biomedical and pharmaceutical applications.

\section{Results and discussion}

\section{Preparation of CNT-stabilized nanostructured lipid particles}

Powdered forms of $-\mathrm{COOH}$ functionalized multi-walled (MW) CNTS, $-\mathrm{OH}$ functionalized MWCNTs and pristine singlewalled (SW) CNTs were pre-dispersed in water by probe ultrasonicator followed by addition of molten Dimodan U (DU). The DU is a commercial lipid mainly consisting of monoglycerides and it forms a bicontinuous cubic Pn $3 \mathrm{~m}$ phase (crystallographic space group 224) in excess water (at room temperature). ${ }^{36}$ The Pn3m phase exhibits 3-dimensionally folded curved bilayer draped over double diamond type mathematical minimal surface ${ }^{15}$ separating two continuous water networks (Fig. 1A). The 'bicontinuous' feature of this nanostructure facilitates reconstitution and practically uninterrupted diffusion of amphiphilic molecules like membrane proteins providing them native like environment. ${ }^{37}$ Each DU-CNT mixture in water was subjected to further ultrasonication in order to break large and inconsistent lipid domains formed during the hydration of a lipid. Dispersing the bulk lipid in this manner apparently speeds up the process of lipid hydration and thus equilibrium formation of self-assembled nanostructures, which otherwise requires considerably long time (could be one or many days) and/or freeze-thaw cycling. While the bulk lipid phase breaks into particles, the lipid coated CNTs are presumed to form a shell around them (Fig. 1B). The interfacial stabilization avoids further aggregation of both, nanostructured lipid particles as well as CNTs. Such kinetic stabilization can be called a Pickering ${ }^{38}$ (due to the use of solid particles) emulsion of oil-in-water $(\mathrm{O} / \mathrm{W})$ type, where lipid forms an 'oil phase' while 'excess water' forms a continuous emulsion medium. ${ }^{28}$ Such an aqueous medium 

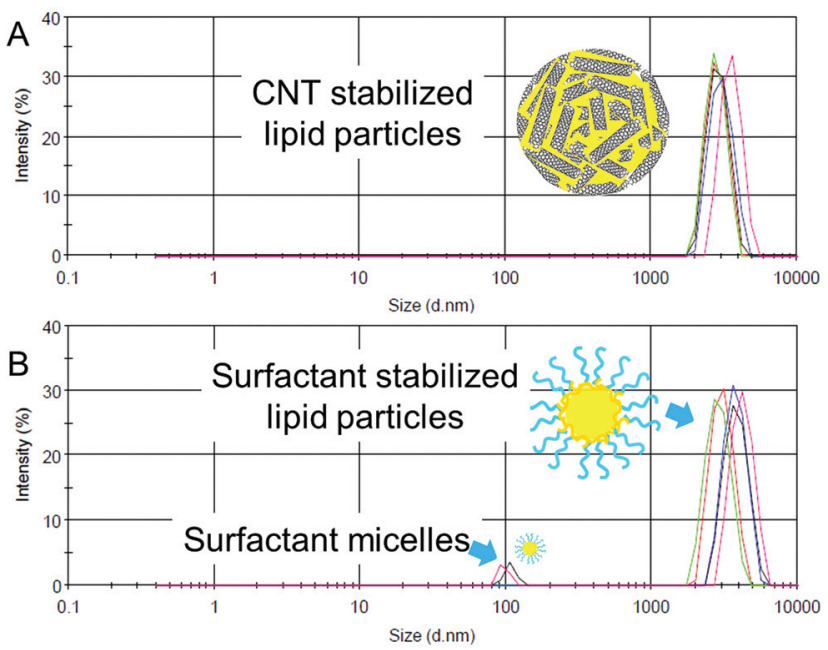

Fig. 2 Particle size of nanostructured lipid particles. (A) An average particle size of CNT stabilized nanostructured lipid particles was in the range of $2-5 \mu \mathrm{m}$ and it was comparable to the size of lipid particles stabilized by pluronic F127 surfactant (B), as determined from dynamic light scattering experiments. An additional peak for surfactant based system could be attributed to surfactant micelles of about $100 \mathrm{~nm}$ size.

and the hydrophobic lipid core of similar lipid particles (stabilized by surfactants) are shown to provide right environments for incorporation of hydrophilic and hydrophobic active pharmaceutical agents, respectively. ${ }^{32,33}$ Moreover, the amphiphilic lipid bilayer fabricating the cubic nanostructure is useful for reconstitution of membrane proteins. ${ }^{39,40}$ Dynamic light scattering studies confirmed that CNT stabilized nanostructured lipid particles were comparable in size $(2-5 \mu \mathrm{m})$ with those stabilized by conventional surfactant stabilizerpluronic F127 (Fig. 2).

\section{Lipid decoration on CNTs revealed by Raman spectroscopy}

Raman spectroscopy revealed CNT-lipid interactions responsible for their mutual stabilization in aqueous solutions (Fig. 3). The CNT spectra contain typical Raman graphite bands; the $\mathrm{G}$ band is assigned to the in-plane vibration of ' $\mathrm{C}-\mathrm{C}$ bond', the D band (not shown) is activated by the presence of disorder in carbon systems and the $\mathrm{G}^{\prime}$ band attributed to the overtone of the D band. ${ }^{41}$ Upon interaction and formation of CNT stabilized lipid particles the $\mathrm{G}$ and $\mathrm{G}^{\prime}$ bands display a blue shift (shift to higher wavenumbers). Table 1 lists the peak centers for characteristic bands from pure CNTs and nanostructured lipid particles containing CNTs. A blue shift, in case of CNTs, is a result of either or a combination of the following: (1) the dispersion of CNTs, as opposed to a bundled state when pure ${ }^{41}$ (2) coating of CNTs; Douroumis et al. ${ }^{42}$ have shown significant blue shifts when analysing lipid coated SWCNTs and suggest that the presence of functionality affects the symmetric radial vibrations of the hollow cylinders through stiffening which are attributed to the interactions between the CNTs and the lipid molecules (3) subjecting CNTs to a high pressure. ${ }^{43}$
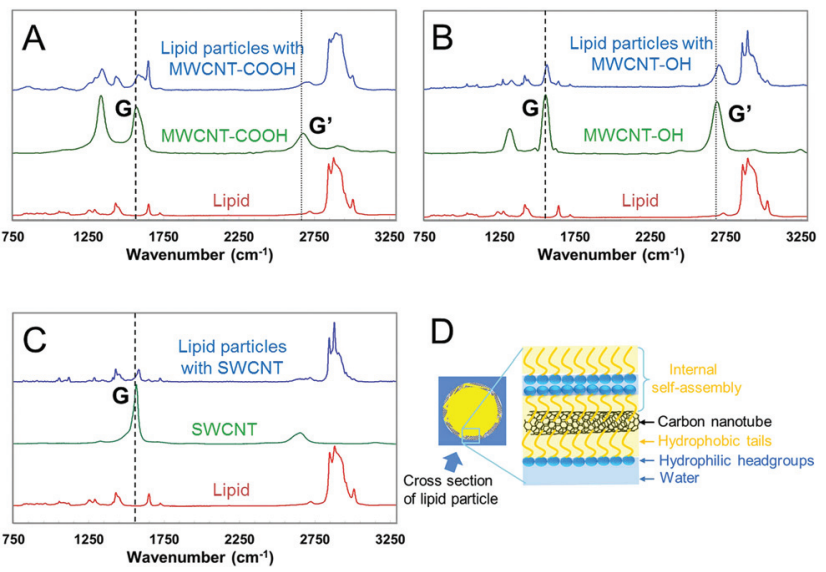

Fig. 3 Raman spectra elucidating lipid decoration on CNTs. Raman spectra of (A) pure lipid, MWCNT-COOH and CNT stabilized lipid nanoparticles containing $5.0 \mu \mathrm{g} \mathrm{ml}^{-1} \mathrm{MWCNT}-\mathrm{COOH}$, (B) pure lipid, $-\mathrm{OH}$ functionalized multi-walled carbon nanotubes (MWCNT-OH) and lipid nanoparticles containing $5.0 \mu \mathrm{g} \mathrm{ml}^{-1} \mathrm{MWCNT}-\mathrm{OH}$, and (C) pure lipid, single-walled carbon nanotubes (SWNT) and lipid nanoparticles containing $0.3125 \mu \mathrm{g} \mathrm{ml}^{-1}$ SWCNT (Other concentrations' Raman spectra are shown in ESI Fig. S1). All curves represent an average of ten spectra where intensities, in arbitrary units (labels not shown) are plotted along $y$-axis. Blue shift in typical G and G' bands of CNTs represent the interaction between CNT surface and lipid molecules. Lipid decoration over CNTs was also depicted by decrease in relative intensities of CNT peaks and appearance of lipid signals which are visible from blue curves. Dashed ( $G$ band) and dotted ( $G$ ' band) lines are used to guide the eyes. (D) Schematic diagram of lipid self-assembling on CNT surface. Alkyl chains of lipid molecules interact with hydrophobic CNT surface whereas the hydrophilic lipid headgroups face aqueous region. Interior of particles is filled with self-assembled lipid nanostructure as indicated by the cross section of lipid particle (yellow).

Table 1 Raman wavenumbers and shift for CNTs and nanostructured lipid particles with CNTs

\begin{tabular}{|c|c|c|c|}
\hline CNT type & $\begin{array}{l}\text { Peak centre } \\
\text { of } \mathrm{CNT}\left(\mathrm{cm}^{-1}\right)\end{array}$ & $\begin{array}{l}\text { Peak centre } \\
\text { of CNT-lipid } \\
\text { nanostructure } \\
\left(\mathrm{cm}^{-1}\right)\end{array}$ & $\begin{array}{l}\text { Wavenumber } \\
\text { shifted }\left(\mathrm{cm}^{-1}\right)\end{array}$ \\
\hline \multirow[t]{2}{*}{ MWCNT-COOH } & 1578.23 (G) & 1600.05 & 21.83 \\
\hline & $2683.72\left(\mathrm{G}^{\prime}\right)$ & 2720.08 & 36.36 \\
\hline \multirow[t]{2}{*}{ MWCNT-OH } & $1570.91(\mathrm{G})$ & 1588.24 & 17.33 \\
\hline & 2687.27 (G') & 2698.18 & 10.91 \\
\hline Pristine SWCNT & $1570.91(\mathrm{G})$ & 1585.45 & 15.44 \\
\hline
\end{tabular}

Significant blue shifts (Table 1) observed in all spectra of lipid nanoparticles containing CNTs provide an evidence for 'dispersed' rather than 'bundled' CNTs. Hydrophobic interactions between CNT surface and alkyl chains of lipid molecules further contribute to the blue shift. Moreover, CNTs are exerted with a greater pressure due to increase in the overall viscosity of lipid particles because of dehydration. Decrease in relative intensities of typical CNT bands (as compared to uncoated CNTs) and emergence of prominent lipid signals also prove the fact that CNTs were decorated with lipid molecules. 
Optimum CNT : lipid ratio and stability of nanostructured lipid particles

As discussed earlier, interface of lipid particles is stabilized by a layer of CNTs enclosing self-assembled nanostructures at the core. However, we observed that there is a particular range of CNT: lipid ratio in which they result in stable emulsions; ratios higher than this cause aggregation of CNTs as shown in Fig. 4 (arrows indicate visible aggregates of excess CNTs). Similarly lower ratios do not lead to stable emulsions because of an insufficient number of CNTs in the solution. Best working concentrations for MWCNT-COOH and MWCNT-OH were found to be between 3 to $5 \mu \mathrm{g} \mathrm{m}{ }^{-1}$ whereas for SWCNT they were in the range of $0.3-0.45 \mu \mathrm{g} \mathrm{ml}^{-1}$. Previous research on the toxicity of pristine MWCNTs demonstrates that $40 \mu \mathrm{g} \mathrm{ml}{ }^{-1}$ dosage should have no toxicity on human T lymphocytes, ${ }^{44}$ while the research on pristine SWCNTs states that $7.5 \mu \mathrm{g} \mathrm{ml} \mathrm{m}^{-1}$ dosage causes only $10 \%$ decrease in cell proliferation and activity in Mesothelioma cell line MSTO-211H. ${ }^{45}$ CNT concentrations employed for stabilizing nanostructured lipid particles, in this work are well below (by an order of magnitude) these toxicity limits. Moreover, the functionalization (in case of MWCNTs) and coating with lipid molecules are expected to enhance biocompatibility and reduce the likelihood of a toxic effect.

We were able to stabilize as low as $2 \%$ dispersed phase emulsions using the same lipid : CNTs ratio (Fig. 5A) used for preparing all above emulsions (5\% dispersed phase). To another end, emulsions up to $20 \%$ lipid material were also prepared by maintaining CNT : lipid ratio constant (Fig. 5A). This wide range of nanostructured lipid particle concentrations facilitates their loading with extensively varied quantities of

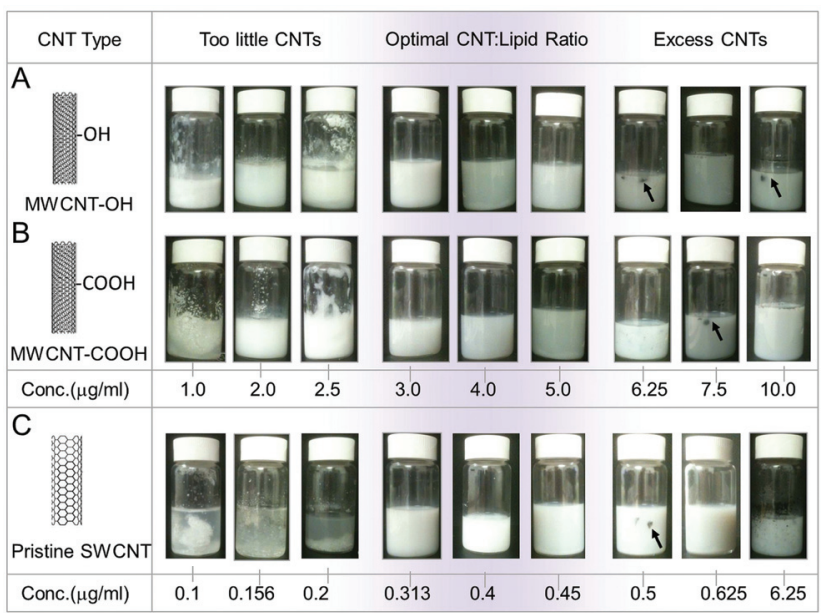

Fig. 4 Optimum CNT concentrations for stable emulsions. Stable emulsions (shown by purple shade) were obtained in the region of 3.0-5.0 $\mu \mathrm{g} \mathrm{ml}^{-1}$ for MWCNT-OH as in (A) and MWCNT-COOH as in (B). The concentration of SWCNTs for stable emulsions as in (C) was about an order of magnitude less than above numbers. It was also possible to get stable emulsions at concentrations very near to these ranges (as indicated by color gradient). For lower concentrations of CNTs, large domains of lipid were seen as there were too little CNTs per lipid whereas for higher concentrations there were too many CNTs which tend to aggregate; some of these are indicated by black arrows.

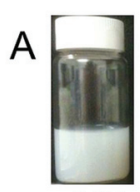

$2.0 \%$

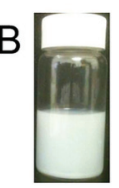

10 minutes

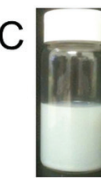

Original

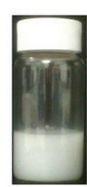

$5.0 \%$

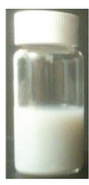

2 days

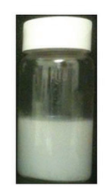

$10.0 \%$

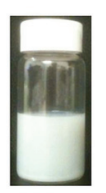

2 months 3 months After shaking

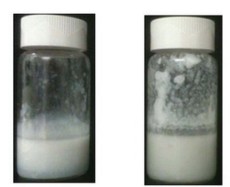

$15.0 \% \quad 20.0 \%$

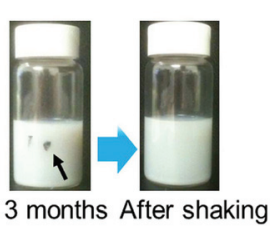

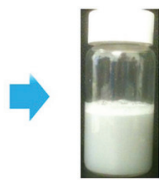

Re-dispersed

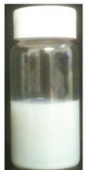

After 2 days
Fig. 5 Stability and regeneration of lipid particulate emulsions. (A) Concentration of dispersed phase, mainly lipid could be tuned from $2 \%$ to $20 \%$. At $20 \%$ the emulsions become thick due to higher lipid material above which it was hard to prepare them using the ultrasonication method. (B) CNT stabilized nanoparticulate emulsions were stable for about 2 months, after which a few CNT aggregates were observed. It was, however possible to re-disperse them merely upon gentle shaking. (C) Emulsions could be completely dehydrated and dispersed again. There was a little foam on the top of re-dispersed emulsion which settled in about 2 days resulting into the stable emulsion. Above studies correspondingly demonstrate interesting properties of CNT stabilized emulsions as, capability of uptaking higher quantities of functional material (e.g. active molecules like drugs), shelf-life and practicability of storage/transport.

drug molecules. CNT stabilized emulsions were stable for about 2 months after which CNTs start aggregating, but they were well dispersed again with only gentle shaking (Fig. 5B). Emulsions were also capable of retaining their stability after complete dehydration via freeze-drying followed by rehydration (Fig. 5C). The dehydrated lipid nanoparticles exhibited lamellar nanostructure similar to the dry lipid at ambient temperatures (see small angles X-ray scattering patterns in ESI Fig. S2†).

\section{Experimental}

\section{Materials}

Single-walled carbon nanotubes (SWCNT) and multi-walled carbon nanotubes with $-\mathrm{OH}$ functionalization (MWCNT-OH) were obtained from Nanoamor (Houston, USA) whereas -COOH functionalized multi-walled CNTs (MWCNT-COOH) were purchased from Sigma-Aldrich (Athens, Greece). The lipid, Dimodan U/J (DU) was a generous gift from Danisco (Brabrand, Denmark). DU is a low-cost commercial product, as compared to its pure lipid analogues, containing more than 98\% mono-glycerides. The triblock copolymer pluronic F127 $\left(\mathrm{PEO}_{99}-\mathrm{PPO}_{67}-\mathrm{PEO}_{99}\right)$ was purchased from Sigma-Aldrich, UK. All solutions were prepared with Millipore water. 


\section{Preparation of nanostructured lipid particles}

First, powdered CNTs (MWCNT-OH, MWCNT-COOH and SWCNT) were dispersed in $100 \mathrm{ml}$ water using probe ultrasonicator (ULT065 from Ultrawave, Cardiff, UK) at 40\% power for 2 minutes without pulse. CNTs were stable on their own (without lipid) during preparation time (for about 20 minutes). The solution of surfactant F127 was prepared by simply stirring $200 \mathrm{mg}$ of surfactant in $100 \mathrm{ml}$ water. $9.5 \mathrm{ml}$ of each of above solutions was then added to $500 \mathrm{mg}$ of molten DU in a glass vial. The mixtures were then subjected to ultrasonication with same parameters as CNT dispersions. Emulsions were allowed to cool down (about 10 minutes) prior to further use.

\section{Particle size measurement using dynamic light scattering}

Particle sizes in dispersions were determined by using the Zetasizer Nano-ZS dynamic light scattering (DLS) equipment (from Malvern Instruments, UK). The data, in the form of particle size distribution was analyzed using Malvern DTS version 5.0. Each sample was tested 10 times and 5 such repeats were performed to get final plots.

\section{Raman spectroscopy on lipid-CNT samples}

CNT, lipid and CNT-lipid samples were dehydrated using nitrogen gas followed by vacuum drying in the desiccator for about 20 minutes. Spectroscopic measurements were carried out on a Horiba Jobin-Yvon LabRAM HR800 spectrometer. A $532 \mathrm{~nm}$ laser was utilized to collect spectra from $100-4000 \mathrm{~cm}^{-1}$ using a grating of $600 \mathrm{~g} \mathrm{~mm}^{-1}$ and blazed at $750 \mathrm{~nm}$. Spectra were acquired using $\times 50$ long working distance objective with a numerical aperture of 0.50 . The confocal hole was set at $100 \mu \mathrm{m}$ for spectral collections. The detector used was an Andor electromagnet (EM) charged coupled device (CCD). A video camera with the Raman system was used to guide spectral collection. All spectra were collected with sample situation on Calcium Fluoride slides (Crystran, UK). The instrumentation was calibrated before operation to silicon at the spectral line of $520.8 \mathrm{~cm}^{-1}$. Spectra were acquired using the $532 \mathrm{~nm}$ laser at $1 \%$ exposure for 10 seconds and accumulated 5 times. Immediate data interrogation and manipulation was carried out on the raw data using the LabSpec 6 spectroscopy software suite (HORIBA Scientific).

\section{Conclusions and perspectives}

To conclude, we were able to produce nanostructured lipid particles with internal self-assembly using various CNTs as emulsion stabilizers. In the past, there have been several studies on using lipids or surfactants for dispersion and functionalization of CNTs, however this is the first report showing a range of CNTs for stabilization of lipid based emulsions. Requirement of very low levels of CNTs is promising for in vivo applications as the amount of CNTs administered into the body is an order of magnitude below toxicity limits revealed by the current literature, in addition, the biocompatibility of CNTs is greatly improved due to their coating with lipid molecules. Furthermore, owing to the possibility of loading functional molecules within lipid self-assembly as well as on CNT surface, the CNT stabilized lipid particles exhibit an enormous potential in emerging areas of pharmaceutical and biomedical sciences with special relevance to the development of combination therapies against major diseases. Fundamental properties of these emulsions are that they exhibit good kinetic stability; moreover, they can be regenerated after total dehydration of constituents. This allows for easy storage and transport of stabilized emulsions for use in harsh environments such as developing countries or forward based military hospitals.

\section{Acknowledgements}

We would like to acknowledge Dr Matija Tomsic, University of Ljubljana, Slovenia for experimental support and reading the manuscript carefully. We also acknowledge the contribution by Prof. Ales Iglic and Mukta Kulkarni, University of Ljubljana, Slovenia regarding purchasing of various carbon nanotubes and hosting NPG for short research visit.

\section{Notes and references}

1 W. Shao, P. Arghya, M. Yiyong, L. Rodes and S. Prakash, Carbon Nanotubes for Use in Medicine: Potentials and Limitations, 2013.

2 H. He, L. A. Pham-Huy, P. Dramou, D. Xiao, P. Zuo and C. Pham-Huy, Bio. Med. Res. Int., 2013, 2013, 12.

3 N. Gulati and H. Gupte, Crit. Rev. Ther. Drug, 2012, 29, 65-88.

4 M. Prato, K. Kostarelos and A. Bianco, Acc. Chem. Res., 2008, 41, 60-68.

5 W. Yang, P. Thordarson, J. J. Gooding, S. P. Ringer and F. Braet, Nanotechnology, 2007, 18, 412001.

6 M. Foldvari and M. Bagonluri, Nanomedicine: Nanotechnology, Biology and Medicine, 2008, 4, 183-200.

7 M. Foldvari and M. Bagonluri, Nanomedicine: Nanotechnology, Biology and Medicine, 2008, 4, 173-182.

8 C. P. Firme Iii and P. R. Bandaru, Nanomedicine: Nanotechnology, Biology and Medicine, 2010, 6, 245-256.

9 N. W. S. Kam, M. O'Connell, J. A. Wisdom and H. Dai, Proc. Natl. Acad. Sci. U. S. A., 2005, 102, 11600-11605.

10 C. Richard, F. Balavoine, P. Schultz, T. W. Ebbesen and C. Mioskowski, Science, 2003, 300, 775-778.

11 C. E. Ashley, E. C. Carnes, G. K. Phillips, D. Padilla, P. N. Durfee, P. A. Brown, T. N. Hanna, J. Liu, B. Phillips, M. B. Carter, N. J. Carroll, X. Jiang, D. R. Dunphy, C. L. Willman, D. N. Petsev, D. G. Evans, A. N. Parikh, B. Chackerian, W. Wharton, D. S. Peabody and C. J. Brinker, Nat. Mater., 2011, 10, 389-397.

12 N. Saito, H. Haniu, Y. Usui, K. Aoki, K. Hara, S. Takanashi, M. Shimizu, N. Narita, M. Okamoto, S. Kobayashi, 
H. Nomura, H. Kato, N. Nishimura, S. Taruta and M. Endo, Chem. Rev., 2014, 114, 6040-6079.

13 Y. Wu, J. S. Hudson, Q. Lu, J. M. Moore, A. S. Mount, A. M. Rao, E. Alexov and P. C. Ke, J. Phys. Chem. B, 2006, 110, 2475-2478.

14 G. Liu and Y. Lin, J. Nanosci. Nanotechnol., 2006, 6, 948953.

15 C. V. Kulkarni, Nanoscale, 2012, 4, 5779-5791.

16 E. Contal, A. Morère, C. d. Thauvin, A. 1. Perino, S. p. Meunier, C. Mioskowski and A. Wagner, J. Phys. Chem. $B, 2010$, 114, 5718-5722.

17 E. J. Wallace and S. P. S. Mark, Nanotechnology, 2009, 20, 045101.

18 A. A. Kapralov, W. H. Feng, A. A. Amoscato, N. Yanamala, K. Balasubramanian, D. E. Winnica, E. R. Kisin, G. P. Kotchey, P. P. Gou, L. J. Sparvero, P. Ray, R. K. Mallampalli, J. Klein-Seetharaman, B. Fadeel, A. Star, A. A. Shvedova and V. E. Kagan, Acs Nano, 2012, 6, 4147-4156.

19 N. Patra and P. Kral, J. Am. Chem. Soc., 2011, 133, 61466149.

20 R. Qiao and P. C. Ke, J. Am. Chem. Soc., 2006, 128, 1365613657.

21 C. Paukner, K. K. K. Koziol and C. V. Kulkarni, Nanoscale, 2013, 5, 8992-9000.

22 C. V. Kulkarni, W. Wachter, G. R. Iglesias, S. Engelskirchen and S. Ahualli, Phys. Chem. Chem. Phys., 2011, 13, 30043021.

23 Z. Almsherqi, F. Margadant and Y. Deng, in Advances in Planar Lipid Bilayers and Liposomes, Elsevier, 2010, vol. 12, ch. 4, pp. 79-99.

24 Ehud M. Landau and Javier V. Navarro, Application: US Pat, US2001/0025791A1, 2001.

25 C. V. Kulkarni, in Advances in Planar Lipid Bilayers and Liposomes, ed. A. Iglic, Academic Press, 2010, vol. 12, ch. 9, pp. 237-272.

26 Y. Song, R. M. Dorin, R. M. Garcia, Y. B. Jiang, H. Wang, P. Li, Y. Qiu, F. van Swol, J. E. Miller and J. A. Shelnutt, J. Am. Chem. Soc., 2008, 130, 12602-12603.
27 J. Gustafsson, H. Ljusberg-Wahren, M. Almgren and K. Larsson, Langmuir, 1996, 12, 4611-4613.

28 C. V. Kulkarni, in Encyclopaedia of Biophysics, ed. G. C. Roberts, Springer Varlag, 2012.

29 A. Yaghmur and O. Glatter, Adv. Colloid Interface Sci., 2009, 147-148, 333-342.

30 C. V. Kulkarni and O. Glatter, in Self-Assembled Supramolecular Architectures: Lyotropic Liquid Crystals, ed. N. Garti, John Wiley \& Sons, Inc., 2012, ch. 6.

31 S. B. Rizwan, B. J. Boyd, T. Rades and S. Hook, Expert Opin. Drug Del., 2010, 7, 1133-1144.

32 T. H. Nguyen, T. Hanley, C. J. Porter, I. Larson and B. J. Boyd, J. Pharm. Pharmacol., 2010, 62, 844-855.

33 K. W. Lee, T. H. Nguyen, T. Hanley and B. J. Boyd, Int. J. Pharm., 2009, 365, 190-199.

34 R. J. Worthington and C. Melander, Trends Biotechnol., 2013, 31, 177-184.

35 H. Araoka, Nihon rinsho. Jpn. J. Clin. Med., 2012, 70, 305310.

36 R. Mezzenga, C. Meyer, C. Servais, A. I. Romoscanu, L. Sagalowicz and R. C. Hayward, Langmuir, 2005, 21, 3322.

37 E. M. Landau and J. P. Rosenbusch, Proc. Natl. Acad. Sci. U. S. A., 1996, 93, 14532-14535.

38 S. U. Pickering, J. Chem. Soc., 1907, 91, 2001.

39 M. Caffrey, Annu. Rev. Biophys., 2009, 38, 29-51.

40 C. V. Kulkarni, A. M. Seddon, O. Ces and R. H. Templer, Soft Matter, 2010, 6, 4339-4341.

41 L. Bokobza and J. Zhang, Express Polym. Lett., 2012, 6, 601.

42 D. Douroumis, D. G. Fatouros, N. Bouropoulos, K. Papagelis and D. Tasis, Int. J. Nanomed., 2007, 2, 761-766.

43 Q. Zhao and H. D. Wagner, Philos. Trans.: Math., Phys. Eng. Sci., 2004, 362, 2407-2424.

44 M. Bottini, S. Bruckner, K. Nika, N. Bottini, S. Bellucci, A. Magrini, A. Bergamaschi and T. Mustelin, Toxicol. Lett., 2006, 160, 121-126.

45 P. Wick, P. Manser, L. K. Limbach, U. Dettlaff-Weglikowska, F. Krumeich, S. Roth, W. J. Stark and A. Bruinink, Toxicol. Lett., 2007, 168, 121-131. 